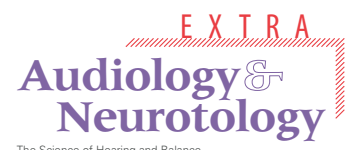

\section{Audiol Neurotol Extra 2014;4:56-61}

DOI: $10.1159 / 000365835$

Publisned onine: August 16, 2014

\title{
Electromagnetic Compatibility of Cochlear Implant with an Aircraft Cockpit
}

\author{
Juliana Maria Araujo Caldeirad Fabio Andrade de Almeida ${ }^{a}$ \\ Marcos Antônio Ribeiro ${ }^{a}$ Jana D'Arc Abdalla Alonso ${ }^{a}$ \\ Maria Valéria Schmidt Goffi-Gomez ${ }^{b}$ Ricardo Ferreira Bento ${ }^{c}$ \\ ${ }^{a}$ Aeronautical Systems Division, Institute of Aeronautics and Space, São José dos Campos, \\ ${ }^{b}$ Cochlear Implant Group, Clinical Hospital and ${ }^{\mathrm{C}}$ Department of Otorhinolaryngology, and \\ dUniversity of São Paulo School of Medicine, São Paulo, Brazil
}

\section{Key Words}

Cochlear implant $\cdot$ Aviation medicine $\cdot$ Aircraft $\cdot$ Hearing loss

\section{Abstract}

Background: For the cochlear implant (CI) to be an option for aircraft pilot rehabilitation, the objective of this study was to rule out the possibility of interference between the implant and the aircraft equipment. Method: All security instruments of the EMB120 aircraft on the ground with the engine running were tested with a bilateral CI user sitting in the copilot's seat. $\boldsymbol{R e}$ sults: The presence of the CI user had no effect on any of the navigation equipment during the test flight. The volunteer reported no discomfort, diminished hearing, headache or neurological symptoms during or after the test flight. Conclusion: There is an electromagnetic compatibility between the EMB120 aircraft instruments and the bilateral CI user in the cockpit.

\section{Introduction}

The cochlear implant (CI) is currently standard rehabilitation equipment for severe sensorineural and profound bilateral hearing loss [Bento et al., 2004; Yamaguchi and Gomez, 2009]. There are currently more than $500.000 \mathrm{CI}$ users worldwide, and an increasing number of implantees experience good hearing rehabilitation [Clark et al., 2014]. This electronic

The study was conducted at the Institute of Aeronautics and Space, Air Force, Brazil. 
device transforms sound stimuli into electrical signals that directly excite the remaining nerve fibers in the cochlea, sending the signals by the auditory nerve to be decoded by the cerebral cortex [Oyanguren et al., 2010]. In recent years, indications for the implant have expanded, and patients with moderate or unilateral hearing loss have received implants [Mlynski and Plontke, 2013; Sampaio et al., 2011].

There has been a great deal of discussion about the significant incidence of hearing loss in civil aviation pilots [Buckey et al., 2001; Kozin, 2005; Lang and Harrigan, 2012; Lindgren et al., 2008; Wagstaff and Arva, 2009]. These professionals, with extensive experience and countless flight hours may be possible candidates for CIs, for example, after an episode of sudden hearing loss at a certain stage of their career. The high cost of training and time spent to gain flight experience are important factors in the continuity of the professional activity of these individuals. However, the effectiveness of radio communication with CI users, with the background noise inherent in the cockpit, still needs to be ascertained.

However, the possibility of interference between the implant and the aircraft should be ruled out because the Cl's transmission of sound stimuli involves radio frequencies and its external component is attached using a magnetic device [Albernaz, 1996]. The July 2012 newsletter of the Federal Aviation Administration (FAA) [2014] explains that due to the popularity of CIs, their use is allowed for passengers during all flight operations. However, there have been no references on regulating their use in aircraft cabins or on assessing the electromagnetic compatibility of the $\mathrm{CI}$ with the safety equipment of the aircraft. Answering these questions is the first step to making it possible for pilots to undergo hearing rehabilitation by means of CIs and use them during flights.

The primary objective of this study was therefore to evaluate the electromagnetic compatibility of bilateral CI use with cockpit and flight instruments in an EMB120 aircraft. The secondary objective was to evaluate the interference of the electromagnetic emissions of the aircraft cockpit instruments with the functioning of the CI.

\section{Materials and Methods}

This was an observational and individual study. The study was approved by the Ethics Committee of the Clinical Hospital, University of São Paulo School of Medicine, under the protocol No. 294.148, and the test was coordinated by the Institute of Aeronautics and Space (IAE) of the Department of Aerospace Science and Technology (DCTA) in São José dos Campos, Brazil.

The study was conducted with the voluntary participation of a user of bilateral Opus 2 implants (MED-EL, Innsbruck, Austria) who read and signed the informed consent form. The female volunteer was 28 years old, with a higher education level, had postlingual deafness and had no symptoms of dizziness or claustrophobia or other symptoms that might make her uncomfortable in the cockpit. She was not carrying any electronic equipment or any other equipment that would emit electromagnetic energy (pacemaker, brain stimulator or other implanted electronic/metal prosthesis) other than her CIs. She exhibited good auditory discrimination for conversational speech, and on a free-field audiometry, the intelligibility test showed results of $90 \%$ accuracy for monosyllables and $100 \%$ accuracy for trisyllables (performed approx. $1 \mathrm{~h}$ before the test in the aircraft). Two other male volunteers, one with a unilateral implant and the other with bilateral Nucleus CIs (Cochlear Limited, Sydney, N.S.W., Australia), who also read and signed the informed consent form, remained seated in the front seats of the aircraft (outside the cockpit) with their implants switched on and charged throughout the aircraft's instrument verification procedure. These volunteers also had good auditory discrimination for conversational speech and were not carrying any electronic equipment other than their CIs that emitted electromagnetic energy. 
The aircraft chosen was the EMB120 because it was a turboprop aircraft that had a higher level of noise in the cabin than aircrafts with jet propulsion and therefore constituted a more demanding sound environment for the implants. In addition, the avionics systems of the EMB120 are based on technology from the late 1970s and early 1980s that produce more electromagnetic emissions and are also more susceptible to electromagnetic interference.

Testing was conducted to ascertain the degree of interference caused by a bilateral CI user sitting in the copilot's seat in the cockpit with the systems necessary for safe navigation. This test was conducted by IAE engineers and flight mechanics. The test was performed in an open environment away from other sources of interference to minimize the influence of the electromagnetic environment on the aircraft's systems, which could invalidate or mask the effects relating to sources of interference within the aircraft itself. The area designated for the test was the courtyard of the VIP lounge of the DCTA runway in São José dos Campos.

The engine starting procedure was carried out according to the flight manual, including procedures to put the aircraft into the 'ready for taxi' mode. The engines remained running with the aircraft on the ground during the test. Communication was maintained with the bilateral implantee seated in the copilot's seat through a VHF radio that was external to the aircraft to ensure that the implant was switched on and fully operational throughout the test. Concomitantly, possible changes in normal aircraft functions were checked using the indicators of the systems described below.

(1) Electricity Generation System: electrical panel warning and readings of the two voltmeters.

(2) Alarm System: readings and warnings of the multiple alarm panel, master and fire warning, and the warning and caution lights of the stall warning panel.

(3) Communication System: interference with VHF radio communication at the frequencies of 124.10 and $121.90 \mathrm{MHz}$.

(4) Navigation System: readings of the radio altimeter, radio/barometric altimeter, standby horizon indicator, attitude director indicator (ADI), distance measuring equipment (DME), global positioning system (GPS), electronic horizontal situation indicator (EHSI), multifunction display (MFD), radio magnetic indicator (RMI), electronic attitude director indicator (EADI) and marker beacon. Possible interference with the radar systems and the right EADI could not be verified because they were in maintenance.

(5) Propulsion System: readings of the interturbine temperature indicators T6, torque indicators, propeller speed indicators $\mathrm{np}$, high- and low-pressure spool indicators $\mathrm{nh} / \mathrm{nl}$, oil temperature and pressure indicators and indicators of the starting/ignition panel and propeller system controls and indicators.

(6) Fuel System: readings of the fuel feed panel, fueling panel and fuel management panel. Interference with the activation of the electric fuel boost pumps and crossfeed valve was also measured.

(7) Flight Command System: interference with the activation of the rudder control panel valves was measured, and we verified the warning of the lemes control system or any movement thereof. Interference with the flaps control system was measured, and we verified the warnings (flap annunciator unit on the flap warning indication panel and flap position indicator) and movements of the flaps. Interference with the autopilot system was measured, and we verified the readings of the autopilot and flight control panels as well as the movements of the flight control surfaces and compensators.

(8) Landing Gear and Brake System: readings of the landing gear panel and brake control panels.

(9) Hydraulic System: interference with the activation of the electric pumps was measured, and we verified the hydraulic power panel. 
(10) Anti-Ice System: readings on the ice/rain protection panel, monitor panel, windshield panel, pitot/static panel, tat panel and ice detector panel.

(11) Air Conditioning System: readings of the air conditioning control panel, pressurization control panel and oxygen control panel.

The three volunteers answered a questionnaire administered before and after the test flight that included questions about any symptoms of abnormal hearing, tinnitus, dizziness or headache before, during and after the test. Free-field audiometry was performed approximately $1 \mathrm{~h}$ before the test flight and was only to be repeated if the volunteers presented any symptoms. The volunteers were also instructed to report any change in hearing in the coming days and were asked 30 days later if they needed to make any adjustments to their implant settings.

\section{Results}

There was no effect on any of the navigation equipment tested during the test flight in the presence of a bilateral CI user in the cockpit sitting in the copilot's seat, as per Test Report RE ASA-S 06/0/2013.

Electromagnetic compatibility between the $\mathrm{CI}$ and the aircraft safety equipment was further confirmed by the presence of the two other volunteers, one with a unilateral implant and the other with a bilateral implant, who remained seated in the front seats of the aircraft and who, although out of the cockpit, were very close to the equipment of the aircraft model tested.

The bilaterally implanted volunteer sitting in the copilot's seat promptly answered all questions asked via the VHF radio system, showing intelligibility similar to a normal hearing person (5/5) and not requiring repetition of the guidelines to understand them via the radio.

The three volunteers reported no discomfort, impaired hearing, headache or neurological symptoms during or after the test flight. Thirty days after the test, they also reported that their hearing had not worsened and they did not need to adjust their implant settings.

\section{Discussion}

Pilots periodically need to renew their Aviation Medical Certificate (CMA), which assesses, among numerous medical conditions, hearing capacity. Under current Brazilian Civil Aviation Regulations, RBAC-2011, pilots with severe hearing loss are prevented from performing their duties because their rehabilitation through the use of a hearing aid is not described. This study evaluates the interface between a bioengineering device and the technology involved in the flight safety of an aircraft. Its importance lies in delivering technical data regarding the use of CIs in cockpit conditions and the potential applicability of the results in civil aviation laws with respect to issuing CMAs. Minimum hearing requirements are among the necessary criteria for issuing a pilot's CMA. These are described in the laws that regulate civil aviation across the world, and there are differences in hearing threshold criteria for pilots' initial validation or revalidation. According to Brazilian legislation, initial pilot candidates must have sufficient hearing in each ear to perceive down to $35 \mathrm{~dB}$ at frequencies of $500-2,000 \mathrm{~Hz}$ and down to $50 \mathrm{~dB}$ at $3,000 \mathrm{~Hz}$. In case of revalidation, a pilot with unilateral deafness can be considered fit if the other ear has the requisite hearing described above. The Civil Aviation Authority of the United Kingdom recognizes that hearing loss is inherent in the pilot's career progression. Experienced pilots who are candidates for revalidation even without fulfilling the necessary minimum hearing criteria may be judged fit if they satisfactorily perform all 
tasks involving hearing in technical proficiency tests. The US legislation (FARS, Code of Federal Regulations) describes the possible use of hearing aids to achieve the required hearing threshold. It also allows for the possibility of candidates with bilateral deafness of undergoing training towards conducting a test flight for a private pilot's license. Passing this stage of technical proficiency allows the pilot restricted authorization, which is limited to areas where radio communication is not required.

However, there is as yet no description in national and international civil aviation laws regarding the possibility of using CIs to meet hearing requirements, i.e., to achieve the minimum thresholds required for pilots' CMA revalidation. The CI may provide hearing rehabilitation for experienced pilots with countless flight hours presenting with bilateral profound sensorineural hearing loss. The high cost of training and gaining flight experience are important factors in the continuity of professional activity of these individuals.

To perform an auditory discrimination test with CI users in a realistic situation involving a noisy aircraft cabin, it was necessary to pretest the electromagnetic compatibility between the $\mathrm{CI}$ and the flight instruments because the transmission of sound stimuli involves radio frequencies and the external component of the $\mathrm{CI}$ is attached using a magnetic device.

The $\mathrm{CI}$ is an electronic prosthesis surgically inserted into the inner ear. Unlike conventional hearing aids, the $\mathrm{CI}$ captures sound waves and generates electrical impulses that directly stimulate the cochlear nerve. It consists of two units, one external, used by the patient at will, which contains a microphone, speech processor and transmitter antenna, and one internal, which consists of a surgically implanted unit that contains a receiver/stimulator and a thin electrode wire. This electrode array is inserted into the cochlea, and the auditory nerve fibers are stimulated at different frequency-coding sites (discrimination). The transcutaneous route of signal transmission from the processor to the implanted electrodes is currently the most used. In this method, the external transmitter encodes the information (sound stimulus) that is transmitted by radio frequencies from the external coil to the internal coil that is implanted in the temporal bone. The decoded signal travels through the electrodes to stimulate the nerve fibers. Transcutaneous attachment of the external coil (receiver) to the internal one (stimulatory) is achieved using a magnetic device [Albernaz, 1996].

This study was conducted at DCTA, Embraer Precursor Department, allowing access to the EMB120 aircraft. It was important to use this aircraft in this study because it was a twinengine turboprop airplane with high noise emission and therefore required more auditory response from the implantees. In addition, the avionics systems of the EMB120 were based on technology from the late 1970s and early 1980s, which emit more electromagnetic emissions and are also more susceptible to this type of interference. The EMB120 was equipped with Pratt \& Whitney engines equipped with Hamilton four-bladed propellers, giving it a maximum cruising speed of $583 \mathrm{~km} / \mathrm{h}$ and a range of 1,482 km. Over 350 EMB120 units are flying worldwide, demonstrating the good characteristics of this regional turboprop for passenger transportation.

The fact that there was no interference between the EMB120 aircraft and the bilateral CI user in the copilot seat suggests the compatibility of a unilateral user with the same model in the cabin of the tested aircraft. However, this study was restricted to one type of aircraft in a grounded situation, with the particular model of CI that was present in the cockpit. Therefore, it has limited generalizability to other types of aircrafts or implants. Regarding the interference of the cabin equipment with the operation of the CI, models from two different brands (Opus 2 of Med-El and Nucleus of Cochlear) were tested and approved, suggesting safety for users of both brands in future tests on the same aircraft.

We conclude that the electromagnetic compatibility between the EMB120 aircraft and the $\mathrm{CI}$ introduced into the cockpit environment of the aircraft met the requirements of the MIL-STD-464 standard [Department of Defense, 1997], showing no changes in the analyzed 
aircraft equipment that could endanger flight safety. There was also no interference in the functioning of the CI due to the cockpit instruments of the aircraft. CIs are now a global reality offering the possibility of hearing rehabilitation for all, including civil aviation pilots. Further studies should therefore be developed to provide technical data for legislation regulating hearing criteria for issuing CMAs. This research will assist in assessing the ability of such professionals to continue their work activity while using CIs.

\section{Disclosure Statement}

The authors have no conflicts of interest to declare.

\section{References}

Albernaz PLM: Cochlear implant. Part 2. Ver Bras Med Otorrinolaringol 1996;3:119-122.

-Bento RF, Neto RB, Castilho AM, Gómez VG, Giorgi SB, Guedes MC: Auditory results with multicanal cochlear implant in patients submitted to cochlear implant surgery at University of São Paulo Medical School - Hospital das Clínicas. Rev Bras Otorrinolaringol 2004;70:632-637.

Brazilian Civil Aviation Regulations. RBAC No. 67, Amendment No. 00; 2011 (in Portuguese). http://www2.anac. gov.br/biblioteca/rbac/RBAC67EMD00.pdf (accessed February 11, 2014).

Buckey JC Jr, Musiek FE, Kine-Schoder R, Clark JC, Hart S, Havelka J: Hearing loss in space. Aviat Space Environ Med 2001;72:1121-1124.

Civil Aviation Authority. Otorhinolaryngology Guidance Material (MED.B.080). http://www.caa.co.uk/default. aspx? catid = 2499\&pagetype = 90\&pageid $=13769$ (accessed February 11, 2014).

Clark GM, Hochmair I, Wilson BS: For the development of the modern cochlear implant - a device that bestows hearing to individuals with profound deafness. http://www.laskerfoundation.org/awards/2013_c_ description.htm (accessed February 11, 2014).

Department of Defense, MIL-STD-464: Electromagnetic environmental effects: requirements for System. Washington DC, Department of Defense, 1997.

Department of Transportation, Federal Aviation Administration: Cochlear implants exempt from rules regarding portable electronic devices. InF0 07022. http://www.faa.gov/other_visit/aviation_industry/airline_operators/airline_safety/info/all_infos/media/2007/inF007022.pdf._Published December 2007 (accessed February 11, 2014).

FARS. Code of Federal Regulations, FARS 14 CFR, Part 67, Medical Standards and Certification. http://www.flightsimaviation.com/data/FARS/ (accessed February 11, 2014).

Kozin OV: Specific features of professional hypoacusis in airline pilots (in Russian). Vestn Otorinolaringol 2005;5: 16-19.

Lang GT, Harrigan MJ: Changes in hearing thresholds as measured by decibels of hearing loss in British Army Air Corps lynx and apache pilots. Mil Med 2012;177:1431-1437.

Lindgren T, Wieslander G, Dammstrom BG, Norback D: Hearing status among commercial pilots in a Swedish airline company. Int J Audiol 2008;47:515-519.

Mlynski R, Plontke S: Cochlear implants in children and adolescents (in German). HNO 2013;61:388-398.

Oyanguren V, Gomes MVG, Tsuji RK, Bento RF, Neto RB: Auditory results from cochlear implants in elderly people (in English, Portuguese). Braz J Otorhinolaryngol 2010;76:450-453.

-Sampaio AL, Araújo MF, Oliveira CA: New criteria of indication and selection of patients to cochlear implant. Int J Otolaryngol 2011;2011:573968.

-Wagstaff AS, Arva P: Hearing loss in civilian airline and helicopter pilots compared to air traffic control personnel. Aviat Space Environ Med 2009;80:857-861.

Yamaguchi CT, Gomez MVSG: Audiologic profile of hearing aid users in the contralateral ear of a cochlear implant: preliminary results (in Portuguese). Rev CEFAC 2009;11:494-498. 